\title{
Extração de cobre e de zinco por soluções multielementares em solos do sul do Brasil
}

\author{
Copper and zinc extracted by multielement solution for Southern Brazilian soils
}

\author{
Leandro Bortolon ${ }^{\mathrm{I}^{*}}$ Clesio Gianello ${ }^{\mathrm{I}}$
}

\begin{abstract}
As quantidades extraídas de cobre $(\mathrm{Cu})$ e de zinco RESUMO (Zn) pelas soluções de $\mathrm{HCl} 0,1 \mathrm{~mol} \mathrm{~L}^{-1}$ ( $\mathrm{HCl}$ ) foram comparadas com as extraídas pelas soluções de Mehlich-1 $\left(M_{1}\right)$ e de Mehlich$3\left(M_{3}\right)$. Foram utilizadas 130 amostras de solo representando as principais áreas agrícolas do Estado do Rio Grande do Sul, as quais foram extraídas do banco de solos do Laboratório de Análises de Solo da Universidade Federal do Rio Grande do Sul. A correlação entre as quantidades extraídas pelos métodos indicou alto grau de associação e de significância. A quantidade de $C u$ extraída pela solução de $M_{3}$, em média, não diferiu do $\mathrm{HCl}$. A quantidade extraída de Zn pela solução de $M_{3}$, entretanto, foi inferior à extraída por $\mathrm{HCl}$. As quantidades extraídas de $\mathrm{Cu}$ e Zn pelas soluções de $\mathrm{HCl}$ e de $\mathrm{M}_{1}$ não diferiram estatisticamente. As soluções de $M_{1}$ e de $M_{3}$ são eficientes para a extração multielementar simultânea de $\mathrm{Cu}$ e $\mathrm{Zn}$ dos solos e são alternativas viáveis para serem utilizadas em laboratórios de análises de solo dos Estados do Rio Grande do Sul e de Santa Catarina.
\end{abstract}

Palavras-chave: análise de solo, Mehlich-1, Mehlich-3.

\section{ABSTRACT}

Copper (Cu) and zinc $(\mathrm{Zn})$ amounts extracted with 0.1 molar $\mathrm{L}^{-1} \mathrm{HCl}(\mathrm{HCl})$ for the most representative soil types of Rio Grande do Sul State (Brazil) were compared with those extracted with Mehlich-1 $\left(M_{3}\right)$ and Mehlich-3 $\left(M_{3}\right)$ solutions. The nutrients' amounts extracted by different methods indicated high correlation degree. The $M_{3}$ solution extracted similar copper amounts than $\mathrm{HCl}$; however, lower amounts of $\mathrm{Zn}$. The $M_{1}$ and $M_{3}$ solutions are efficient for simultaneous multielement extraction and can be used in soil testing laboratories for RS and SC states.

Key words: soil test, Mehlich-1, Mehlich-3.
A utilização de soluções extratoras multielementares está sendo estudada por diversos pesquisadores com o objetivo de simplificar os procedimentos analíticos, baixar os custos de análise e testar a extração simultânea de vários elementos. Concomitantemente, métodos de determinação multielementares estão sendo utilizados em vários laboratórios, para aumentar a exatidão analítica, diminuir os limites de detecção e determinar vários elementos (MALLARINO, 2003).

A solução extratora denominada Mehlich-3 $\left(\mathrm{M}_{3}\right)$ tem sido uma das mais estudadas para essa finalidade (MALLARINO, 2003); entretanto, para a sua utilização nos laboratórios de análise de solo, deve ser feita a calibração dos valores obtidos pelo método com o rendimento das culturas numa determinada região. No Estado do Rio Grande do Sul, foi conduzido um estudo de calibração dessa solução para fósforo e potássio em que se observou a sua adequabilidade para avaliar a disponibilidade desses nutrientes às plantas, com tabela de interpretação dos valores analíticos dos teores desses nutrientes no solo (SCHLINDWEIN \& GIANELLO, 2008; BORTOLON \& GIANELLO, 2008).

A possibilidade de uso da solução $M_{3}$ para a extração de outros elementos foi pouco estudada em solos do Estado do Rio Grande do Sul. Um estudo feito por BORTOLON (2005) indicou a viabilidade de uso em laboratórios de análise de solo para extração simultânea de fósforo (P), potássio (K), cálcio (Ca) e magnésio (Mg). Além disso, as quantidades de $\mathrm{Cu}$ e

IDepartamento de Solos, Universidade Federal do Rio Grande do Sul (UFRGS), Av. Bento Gonçalves, 7712, 91540-000, Porto Alegre, RS, Brasil. E-mail: leandro.bortolon@ufrgs.br. *Autor para correspondência. 
Zn extraídos por essa solução tiveram relação com as quantidades acumuladas pelas plantas em nove solos do Estado do Rio Grande do Sul. Essa constatação indica que a solução de $\mathrm{M}_{3}$ poderia substituir as soluções extratoras atualmente em uso, conforme feito em outros locais (GARTLEY et al., 2002; GARCIA et al., 1997).

Os laboratórios de análises de solo dos Estados do Rio Grande do Sul e de Santa Catarina utilizam a solução de Mehlich-1 (M), para a extração de $\mathrm{P}$, e de $\mathrm{K}, \mathrm{HCl}$ 0,1 $\mathrm{mol} \mathrm{L}^{-1}$, para a extração de $\mathrm{Cu}$ e de $\mathrm{Zn}$, e KCl 1,0mol L-1 , para a extração de Ca, Mg, Mn e Al trocáveis (TEDESCO et al., 1995). A solução M é também utilizada em outros Estados para a extração de $\mathrm{Cu}, \mathrm{Zn}, \mathrm{Mn}$ e Fe. Originalmente essa solução foi proposta para extração simultânea de P, K, Ca, Mg, Zn e Mn (MEHLICH, 1953). O uso de diferentes soluções para a extração de grupos de elementos reduz a eficiência dos laboratórios de análises de solo e aumenta o custo das análises. Essa limitação pode ser resolvida implementando soluções extratoras multielementares. Para solos do Rio Grande do Sul, há estudos de calibração de $\mathrm{P}$ e $\mathrm{K}$ a campo para a solução de $\mathrm{M}_{3}$ e tabelas específicas para interpretação dos resultados analíticos desses elementos, facilitando a implementação desse método nos laboratórios de análises de solo (SCHLINDWEIN \& GIANELLO, 2008; BORTOLON \& GIANELLO, 2008).

O presente trabalho teve por objetivos verificar a capacidade extrativa das soluções $\mathrm{M}_{1}$ e $\mathrm{M}_{3}$ para Cu e Zn e correlacionar as quantidades extraídas com as determinadas pela solução de $\mathrm{HCl}$ utilizada como método padrão em laboratórios de análise de solos dos estados do Rio Grande do Sul e de Santa Catarina.

O estudo foi conduzido no Laboratório de Análises de Solo da Universidade Federal do Rio Grande do Sul (UFRGS), com amostras de solos das classes Latossolo, Argissolo, Cambissolo, Planossolo, Neossolo, Nitossolo, Vertissolo e Chernossolo, provenientes do estado do Rio Grande do Sul. Essas amostras foram obtidas do banco de solos do Laboratório de Análises de Solo da UFRGS, num total de 130 amostras. A amplitude de variação dos valores de $\mathrm{pH}$ e dos teores de matéria orgânica e de argila das amostras é apresentada na tabela 1 .

Os teores de $\mathrm{Cu}$ e $\mathrm{Zn}$ dos solos foram extraídos pela solução de $\mathrm{HCl}\left(\mathrm{HCl} 0,1 \mathrm{~mol} \mathrm{~L}^{-1}\right.$; pH 1,2) na relação solo:solução de 1:4, com agitação por 30 minutos em agitador horizontal a 120 oscilações por minuto e decantação por 16 horas, conforme metodologia descrita por TEDESCO et al. (1995). A extração com a solução de $\mathrm{M}\left(\mathrm{HCl} 0,05 \mathrm{~mol} \mathrm{~L}^{-1}+\mathrm{H}_{2} \mathrm{SO}\right.$ $\left.0,0125 \mathrm{~mol} \mathrm{~L}^{-1} ; \mathrm{pH} 1,2\right)$ foi feita na relação solo:solução de 1:10, com agitação por cinco minutos em agitador horizontal a 120 oscilações por minuto e decantação por 16 horas, conforme metodologia descrita por TEDESCO et al. (1995). Esses nutrientes foram também extraídos pela solução de $\mathrm{M}_{3}\left(\mathrm{CH}_{3} \mathrm{COOH} 0,2 \mathrm{~mol} \mathrm{~L}^{-1}+\right.$ $\mathrm{NH}_{4} \mathrm{NO}_{3} 0,25 \mathrm{~mol} \mathrm{~L}^{-1}+\mathrm{NH}_{4} \mathrm{~F} 0,015 \mathrm{~mol} \mathrm{~L}^{-1}+\mathrm{HNO}_{3}$

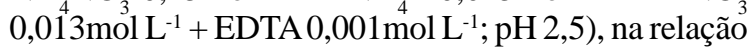
solo:solução de 1:10, com agitação por cinco minutos em agitador orbital a 220rpm. A filtração dos extratos, proposta por MEHLICH (1984), foi substituída pela decantação por 16 horas.

Nos extratos, o $\mathrm{Cu}$ e o $\mathrm{Zn}$ foram determinados por espectrofotometria de absorção atômica. Foi feita a análise de correlação entre as quantidades extraídas dos nutrientes pelas soluções. As médias entre as quantidades de nutrientes extraídas pelas soluções foram comparadas pelo teste t, tendo como padrão para comparação a solução de $\mathrm{HCl}$. Foram estabelecidas regressões lineares entre os teores extraídos pelos métodos, adotando-se o procedimento de passar pela origem, com a finalidade de estabelecer um fator de conversão de valores, conforme descrito por GARTLEY et al. (2002). A significância foi avaliada pelo valor $P$.

Na tabela 1, são apresentados os teores máximos, mínimos, a média e a mediana das diferentes determinações. Os valores obtidos situam-se nas faixas normalmente encontradas em solos agrícolas. As quantidades de $\mathrm{Cu}$ extraído do solo por $\mathrm{HCl}$ não diferiram das extraídas pela solução $\mathrm{M}_{1}$ (Tabela 1) $(\mathrm{P}<0,05)$. A correlação entre as quantidades de $\mathrm{Cu}$

Tabela 1 - Amplitude dos valores de pH e teores de argila e de matéria orgânica de 130 amostras de solos utilizadas e quantidades de cobre e zinco extraídas pelas soluções extratoras $\mathrm{HCl}$, Mehlich 1 e Mehlich 3.

\begin{tabular}{|c|c|c|c|c|c|c|c|c|c|}
\hline \multirow{2}{*}{ Parâmetros } & \multirow{2}{*}{$\mathrm{pH}^{1}$} & \multirow{2}{*}{ Argila } & \multirow{2}{*}{$\mathrm{MO}^{2}$} & \multicolumn{3}{|c|}{------------------Cobre------------------ } & \multicolumn{3}{|c|}{-----------------Zinco----------------- } \\
\hline & & & & $\mathrm{HCl}$ & $\mathrm{M}_{1}$ & $\mathrm{M}_{3}$ & $\mathrm{HCl}$ & $\mathrm{M}_{1}$ & $\mathrm{M}_{3}$ \\
\hline & & \multicolumn{2}{|c|}{---------- $\mathrm{g} \mathrm{dm}^{-3}$---------- } & \multicolumn{6}{|c|}{ 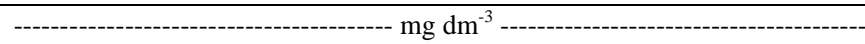 } \\
\hline Mínimo & 4,8 & 30 & 5 & 0,2 & 0,2 & 1,5 & 0,7 & 0,1 & 1,8 \\
\hline Maximo & 6,5 & 700 & 181 & 18,4 & 16,2 & 18,0 & 7,8 & 9,3 & 4,7 \\
\hline Media & 5,6 & 300 & 30 & 4,6 & 5,2 & 5,2 & 4,4 & 4,5 & 4,0 \\
\hline Mediana & 5,6 & 250 & 26 & 3,5 & 3,9 & 4,0 & 4,3 & 4,6 & 3,9 \\
\hline
\end{tabular}

${ }^{1}$ - Determinações efetuadas conforme a metodologia descrita por TEDESCO et al. (1995). 2 - MO: matéria orgânica; HCl: HCl 0,1mol L"1; $\mathrm{M}_{1}$ : Mehlich-1; $\mathrm{M}_{3}$ : Mehlich-3. 
extraído do solo pela solução de $\mathrm{HCl}$ e a solução $\mathrm{M}_{1}$ é mostrada na figura $1 \mathrm{a}$, apresentando um coeficiente de correlação de 0,60 $(\mathrm{P}<0,05)$. A dispersão dos pontos pode ser atribuída à grande variação nas características físicas, químicas e mineralógicas dos solos utilizados. Os dados obtidos corroboram os relatados por LANTMANN \& MEURER (1982) para solos do Rio Grande do Sul; no entanto, o tempo de agitação utilizado por esses pesquisadores foi de 30 minutos. PEREIRA et al. (2001), comparando essas duas soluções em 103 amostras superficiais de solos do Estado do Rio de Janeiro, determinaram um coeficiente de correlação entre ambas de 0,75 , e as quantidades extraídas foram aproximadamente $25 \%$ menores com a solução $\mathrm{M}_{1}$, e o tempo de agitação das amostras foi o mesmo do presente trabalho.
As quantidades extraídas de Cu pela solução $M_{3}$ não diferiram das extraídas com HCl (Tabela 1), provavelmente em razão da composição química da solução de $\mathrm{M}_{3}$, pois nesta é incluído o reagente EDTA que apresenta alta capacidade quelante de cobre (MEHLICH, 1984). No processo de extração, o Cu da solução é complexado, ocorrendo um reequilíbrio químico entre este e o cobre retido na fração sólida do solo, principalmente da fração orgânica. A correlação entre as quantidades de $\mathrm{Cu}$ extraído pelas soluções de $\mathrm{HCl}$ e $\mathrm{M}_{3}$ é mostrada na Figura $1 \mathrm{~b}$, apresentando alto grau de correlação $(r=0,91 ; P<0,01)$. A dispersão dos pontos na regressão é menor do que a observada na comparação entre os extratores $\mathrm{HCl}$ e $\mathrm{M}_{1}$.

A correlação entre as quantidades de $\mathrm{Zn}$ extraído pelas soluções de $\mathrm{HCl}$ e $\mathrm{M}_{1}$ é mostrada na

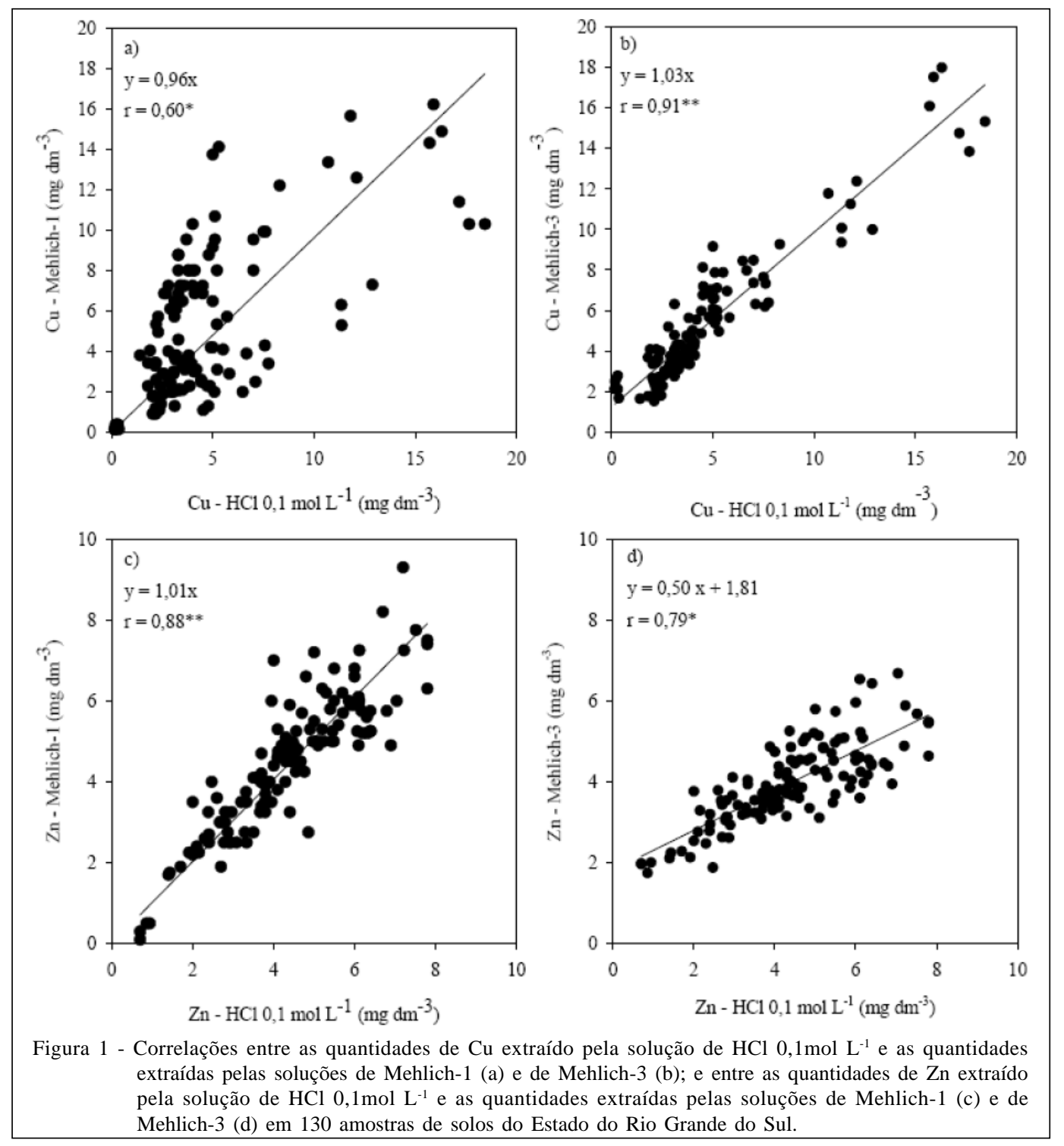

Ciência Rural, v.40, n.3, mar, 2010. 
figura 1c, $(\mathrm{r}=0,88 ; \mathrm{P}<0,01)$, na qual observa-se que estas e não diferiram entre si (Tabela 1$)$, $(\mathrm{P}<0,05)$. Os resultados obtidos neste trabalho corroboram aqueles obtidos por LANTMANN \& MEURER (1982). Esses autores, para a mesma comparação de extratores, obtiveram um coeficiente de correlação de 0,73 num estudo com 10 solos do Estado do Rio Grande do Sul, sem adição de calcário e de Zn. Apesar de ter sido obtido menor coeficiente de correlação, as quantidades extraídas pela solução de $M_{1}$ foram, aproximadamente, $50 \%$ menores que as extraídas pela solução de $\mathrm{HCl}$. Após a aplicação do calcário (para atingir pH 6,0), as soluções extraíram menores quantidades de zinco, possivelmente em razão da maior adsorção deste pelos solos, das reações das soluções extratoras com carbonatos e/ou bicarbonatos livres e do menor tempo de extração (MEHLICH, 1953). Semelhantemente, PEREIRA et al. (2001), num estudo com 103 amostras de solos do Estado do Rio de Janeiro, obtiveram um coeficiente de correlação de 0,87 entre as quantidades extraídas de Zn pelas mesmas soluções. No entanto, a solução $\mathrm{M}_{1}$ extraiu menos $\mathrm{Zn}$ que a solução de $\mathrm{HCl}$.

A correlação entre as quantidades de $\mathrm{Zn}$ extraído dos solos pelas soluções de $\mathrm{HCl}$ e $\mathrm{M}_{3}$ é mostrada na figura $1 \mathrm{~d}(\mathrm{r}=0,79 * *)$. As quantidades de Zn extraído pela solução $M_{3}$ foram, em média, $50 \%$ menores do que as extraídas com a solução de $\mathrm{HCl}$ (Tabela 1), apresentando diferenças significativas $(\mathrm{P}<0,05)$. No entanto, a equação de ajuste da reta não passa pela origem, dificultando o estabelecimento de um fator de conversão de valores. Como os teores críticos de Cu e Zn são muito baixos $\left(<0,5 \mathrm{mg} \mathrm{dm}^{-3}\right)$ nos solos do Estado do Rio Grande do Sul (SBCS/CQFS, 2004) e, em geral, os teores desses nutrientes estão acima desses valores em solos agrícolas, o uso de fatores de conversão pode ser uma alternativa viável para facilitar a implementação de soluções multielementares nos laboratórios de análises de solo, sendo alternativa para o aumento da eficiência e a redução de custos nas análises.

As soluções de Mehlich-1 e de Mehlich-3 são eficientes para a extração multielementar simultânea de $\mathrm{Cu}$ e $\mathrm{Zn}$ dos solos e estas são alternativas viáveis para serem utilizadas em laboratórios de análises de solo dos Estados do Rio Grande do Sul e de Santa Catarina, adotando um fator de conversão de valores para o Zn extraído por Mehlich-3.

\section{AGRADECIMENTOS}

Ao Conselho Nacional de Desenvolvimento Científico e Tecnológico (CNPq), pela concessão de bolsa de pesquisa ao autor Leandro Bortolon.

\section{REFERÊNCIAS}

BORTOLON, L. Métodos de avaliação da disponibilidade de nutrientes para as plantas em solos do Rio Grande do Sul. 2005. 111f. Dissertação (Mestrado em Ciência do Solo) - Programa de Pós-graduação em Ciência do Solo, Faculdade de Agronomia, Universidade Federal do Rio Grande do Sul, Porto Alegre, RS.

BORTOLON, L.; GIANELLO, C. Interpretação de resultados analíticos de fósforo pelos extratores Mehlich-1 e Mehlich-3 em solos do Rio Grande do Sul. Revista Brasileira de Ciência do Solo, Viçosa, v.32, n. spe, p.2751-2756, 2008. Disponível em: <http://www.scielo.br/scielo.php?script=sci_arttext\&pid=S010006832008000700019\&lng=pt\&nrm=iso $>$. ISSN 0100-0683. Acesso em: 21 dezembro 2009 doi: 10.1590/S010006832008000700019 .

GARCIA, A. et al. Comparison of soil tests to determinate micronutrients status in Argentina soils. Communications in Soil Science and Plant Analysis, New York, v.28, p.17771792, 1997.

GARTLEY, K.L. et al. Comparison of soil test extractants used in mid-Atlantic United States. Communications in Soil Science and Plant Analysis, New York, v.33, p.873895, 2002.

LANTMANN, A.F.; MEURER, E.J. Estudo da eficiência de extratores para avaliação da disponibilidade do zinco disponível do solo para o milho. Revista Brasileira de Ciência do Solo, Viçosa, v.6, p.131-135, 1982.

MALLARINO, A.P. Field calibration for corn of the Mehlich3 soil phosphorus test with colorimetric and inductively-coupled plasma emission spectroscopy determination methods. Soil Science Society of America Journal, v.67, p.1928-1934, 2003.

MEHLICH, A. Determination of $\mathbf{P}, \mathrm{Ca}, \mathrm{Mg}, \mathbf{K}, \mathrm{Na}$ and NH4 by North Carolina soil testing laboratories. Raleigh: University of North Carolina, 1953. 4p.

MEHLICH, A. Mehlich 3 soil test extractant: A modification of Mehlich 2 extractant. Communications in Soil Science and Plant Analysis, New York, v.15, p.1409-1416, 1984.

PEREIRA, M.G. et al. Comparação de métodos de extração de cobre, zinco, ferro e manganês em solos do Rio de Janeiro. Revista Brasileira de Ciência do Solo, Viçosa, v.25, p.655660, 2001.

SCHLINDWEIN, J.A.; GIANELLO, C. Calibração de métodos de determinação de fósforo em solos cultivados sob sistema plantio direto. Revista Brasileira de Ciência do Solo, Viçosa, v.32, n.5, p.2037-2049, 2008. Disponível em: <http:/ /www.scielo.br/scielo.php?script=sci_arttext\&pid=S0100 06832008000500025\&lng=pt\&nrm=iso $>$. ISSN 0100-0683. Acesso em: 21 dezembro 2009 doi: 10.1590/S010006832008000500025 .

SOCIEDADE BRASILEIRA DE CIÊNCIA DO SOLO/ COMISSÃO DE QUÍMICA E FERTILIDADE DO SOLO. Manual de adubação e de calagem para os Estados do Rio Grande do Sul e de Santa Catarina. 10.ed. Porto Alegre, 2004. 400p.

TEDESCO, M.J. et al. Análise de solo, plantas e outros materiais. 2.ed. Porto Alegre: Universidade Federal do Rio Grande do Sul, Departamento de Solos, 1995. 174p. 